\title{
El aprendizaje organizacional y su impacto con el rendimiento empresarial y el mercado: el caso del Ecuador
}

Organizational Learning and its Impact on Business Performance as Well as in the Market: the case of Ecuador

A aprendizagem organizacional e seu impacto no rendimento empresarial e no mercado: o caso do Equador

\author{
Gustavo Gallo-Mendoza \\ Universidad Andina Simón Bolívar (UASB). Quito, Ecuador \\ gustavogallo@msn.com \\ https://orcid.org/0000-0001-9465-0415
}

DOI: https://doi.org/10.32719/25506641.2020.8.3

Recibido: 24 de enero de 2020 - Revisado: 31 de enero de 2020

Aceptado: 16 de marzo de 2020

Artículo de investigación

Licencia Creative Commons

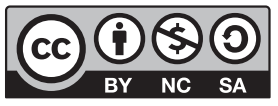




\section{Resumen}

El estudio explica la relación de dos capacidades empresariales: la orientación al mercado y su influencia en la orientación al aprendizaje y, ambos, en los resultados organizacionales de las pequeñas y medianas empresas (pymes) en Ecuador. Esta investigación deductivo-hipotética, de carácter transversal y cuantitativo. El modelo teórico y las hipótesis propuestas fueron contrastadas mediante la aplicación de análisis factorial confirmatorio de las escalas y un análisis basado en el modelo de ecuaciones estructurales. Se basó en el modelo de Sinkula, Baker y Noordewier. Se determinó que sí existe un vínculo entre la orientación al mercado y el aprendizaje, lo cual se traduce en un impacto directo y positivo con los resultados organizacionales, que si bien no alcanzan niveles significativos en el desarrollo de las pymes ecuatorianas, no debe sustentarse dicho resultado en falta o deficiencia de creatividad e iniciativa personal, sino en la actual cultura empresarial existente, la cual debe priorizar estudios de mercado y actividades de desarrollo investigativo de nuevos productos/servicios.

Palabras clave: Mercadeo, aprendizaje, resultados organizacionales, ventaja competitiva, pequeñas y medianas empresas.

JEL: D83 Búsqueda, aprendizaje, información y conocimiento; comunicación; creencia; desconocimiento; M31 Marketing.

\section{Summary}

The study explains the relationship between two business capacities: market orientation and its influence on learning orientation and, both, on the organizational results of small and medium-sized enterprises (SMEs) in Ecuador. This is a transversal and quantitative deductive-hypothetical investigation. The theoretical model and the proposed hypotheses were tested by applying confirmatory factor analysis of the scales and an analysis based on the structural equations model. It was based on the model of Sinkula, Baker and Noordewier. It was determined that there is a link between market orientation and learning, which translates into a direct and positive impact with organizational results, which, although they do not reach significant levels in the development of Ecuadorian SMEs, this result should not be sustained in lack or deficiency of creativity and personal initiative, but in the current existing business culture, which must prioritize market studies and research development activities of new products/services.

Keywords: Marketing, learning, organizational results, competitive advantage, small and medium enterprises.

JEL: D83 Search, learning, information and knowledge; communication; belief; ignorance; M31 Marketing.

\section{Resumo}

O estudo explica a relação de duas capacidades empresariais: a orientação ao mercado e sua influência na orientação à aprendizagem e a influência de ambas sobre os resultados organizacionais das pequenas e médias empresas (PME) no Equador. Esta pesquisa é 
do tipo hipotética-dedutiva, de caráter transversal e quantitativo. O modelo teórico e as hipóteses propostas foram contrastados através da aplicação de uma análise fatorial confirmatória das escalas e uma análise baseada no modelo de equações estruturais. Baseouse no modelo de Sinkula, Baker e Noordewier. Determinou-se que existe um vínculo entre a orientação ao mercado e a aprendizagem, o que se traduz em um impacto direto e positivo sobre os resultados organizacionais. Embora estes não alcancem níveis significativos no desenvolvimento das PME equatorianas, tal resultado não deve justificar-se em falta ou deficiência de criatividade e iniciativa pessoal, devendo-se mais bem à atual cultura empresarial existente, a qual deve priorizar estudos de mercado e atividades de desenvolvimento investigativo de novos produtos/serviços.

Palavras-chave: Comércio, aprendizagem, resultados organizacionais, vantagem competitiva, pequenas e médias empresas.

JEL: D83 Pesquisa, aprendizagem, informação e conhecimento; comunicação; crença; desconhecimento; M31 Marketing.

\section{Introducción}

E 1 estudio explica la relación de dos habilidades empresariales: i) la orientación al mercado; y, ii) la orientación al aprendizaje y su importancia en los resultados de las pymes ecuatorianas. Con el objetivo de que este tipo de organizaciones ya establecidas o nuevas logren un rendimiento organizacional competitivo en el Ecuador.

Se ha analizado la influencia de estas capacidades empresariales claves para los primeros años de operaciones. Dentro de los factores del fracaso de las empresas está su escasa aplicación del conocimiento. Por otra parte, la adquisición y diseminación del conocimiento entre los miembros de la organización y estar abierto a nuevas opiniones, favorece la detección y explotación de oportunidades en las empresas de nueva creación y, por consiguiente, aumenta la probabilidad de supervivencia y crecimiento. De esta manera, Hebel (2007) ha destacado que el aprendizaje organizativo es el puente que une el pensamiento y la acción, capacidad que es vital para que las nuevas empresas traduzcan sus planes de negocio y estrategias a acciones concretas para dar respuesta rápida que efectivice el dinamismo del entorno.

Con los antecedentes antes expuestos, en este estudio se incluyen -además del modelo de la orientación al aprendizaje- algunas investigaciones como las de Morgan y Strong (1998), Baker y Sinkula (1999), Hebel (2007), 
Farrel y Oczkowski (2002), que han considerado a esta capacidad como una rutina organizacional de alto nivel por sus características de generación de conocimiento para aprovechar oportunidades de mercado.

\section{Planteamiento y justificación del problema}

La creación, estabilidad y crecimiento de las pymes ecuatorianas son fundamentales para compensar el cierre de otras empresas y la competencia proveniente de otras economías (Tinajero 2015). Desde 2014, un 9\% de los emprendedores declararon haber cerrado un negocio en los últimos doce meses, cifra que se muestra por encima de lo observado entre los fundadores de negocios establecidos (7\%) y de los no emprendedores (4\%). Pero no es solamente el cierre de empresas lo que afecta negativamente a la actividad empresarial; la dificultad e incertidumbre naturales del proceso emprendedor se evidencia por la tasa de actividad emprendedora temprana que ha sido superior a la tasa de negocios establecidos en los últimos años; el estudio del Global Entrepreneurship Monitor (GEM 2019) para el período (2015-2019) ha mencionado que el porcentaje de los emprendedores que cerraron sus negocios bajó al 7\%, es decir, cada año es peor.

Autores como Jácome y King (2013), los informes GEM (2012-2019), FLACSO (2013), Kantis, Ishida y Komori (2002) han señalado que la mayoría de las nuevas pymes fracasan en los primeros años debido a su inicio de operaciones; las pérdidas financieras, económicas y sociales por este fallo empresarial son significativas, por tanto, es valioso desarrollar modelos o investigaciones que apoyen a las nuevas empresas ecuatorianas.

Este estudio busca generar una herramienta, no solo para que las nuevas pymes ecuatorianas logren operar en el mercado a largo plazo, debido a que se desarrollará una competencia orientada al mercado y al aprendizaje; generarán resultados más eficientes $\mathrm{y}$, por ende, una mayor ventaja competitiva. Los resultados también se aplicarían al conjunto de empresas de todos los sectores productivos ya establecidos que deseen mejorar sus resultados.

El objetivo de la investigación es explicar el impacto que tiene la orientación al mercado y su influencia directa sobre la capacidad organizacional, como es la orientación al aprendizaje. Su aplicación es en el sector producti- 
vo ecuatoriano de alimentos y bebidas. Conjuntamente, se plantean objetivos específicos como: analizar la influencia en el grado de orientación al mercado desarrollado por las empresas; la relación entre el aprendizaje empresarial y el rendimiento organizacional, ajustado a las empresas ecuatorianas nuevas o ya establecidas; y comprobar los efectos de la orientación al mercado y su influencia directa en la variable del aprendizaje organizacional que actúa como moderadora, y su impacto positivo en los resultados de las pymes basadas en economías de eficiencia. ${ }^{1}$

\section{Revisión de literatura}

\section{Las capacidades empresariales}

El conocimiento del mercado es el origen de la estrategia de las nuevas pymes ecuatorianas, la orientación al mercado -como un antecedente que guiará la orientación al aprendizaje- no simultáneo o independiente, como lo han propuesto Hult, Hurley y Knight (2004). Esta orientación está compuesta por la generación de inteligencia de mercado, que consiste en la recolección de información acerca de los consumidores y competidores, así como su difusión y la capacidad de respuesta organizativa con base en esa inteligencia.

Por otro lado, la orientación al aprendizaje es esencial para sobrevivir en ambientes competitivos, es el corazón para la adaptación rápida de los ambientes, es la llave para identificar oportunidades y explotarlas rápidamente y dar mayor valor a los inversionistas. Una empresa que aprende, aplica su conocimiento más rápido. La mejor manera de que las organizaciones sean competitivas es aprender más rápido que sus competidores; rápido significa más eficiencia en aprendizaje, pocos pasos para conseguir usarlo de una manera más sistemática y balanceada en la evaluación de alternativas de acción.

1. Las fases se componen de economías basadas en: i) factores; ii) eficiencia; y, iii) innovación. La economía basada en factores está dominada por la agricultura de subsistencia y negocios de extracción, con una fuerte dependencia en la mano de obra (no calificada) y los recursos naturales. La economía de eficiencia es más competitiva, con procesos de producción más eficientes y una mejor calidad de producto. Las economías de innovación son más intensivas en conocimiento y el sector de servicios se expande (GEM 2016, 13). 
Ante los desafíos actuales en el contexto empresarial ecuatoriano, se considera prioritario para sobrevivir que la empresa sea capaz de aprender, a fin de desarrollar las capacidades internas para hacer frente a la competencia. La orientación al aprendizaje tiene que ver con el entendimiento de la causa y efectos de sus acciones, el cuestionamiento de sus rutinas establecidas y el propósito de la empresa.

\section{La orientación al aprendizaje}

La forma más efectiva y eficiente para crear los comportamientos necesarios para crear un valor superior es la orientación al mercado, entendida como una cultura organizativa que favorece dichos comportamientos. El dinamismo y turbulencia del mercado exige cada vez más que las empresas respondan más adecuadamente a las necesidades de los clientes y consumidores en sus mercados objetivos.

Otro de los factores en el fracaso de las nuevas empresas ha sido su escasa aplicación del conocimiento; la adquisición y diseminación del conocimiento entre los miembros de la organización y estar abierto a nuevas opiniones, favorece la detección y explotación de oportunidades en las empresas de nueva creación $\mathrm{y}$, por consiguiente, aumenta la probabilidad de supervivencia y crecimiento. De esta manera, Hebel (2007) ha destacado que el aprendizaje organizativo es el puente que une el pensamiento y la acción, esta capacidad es vital para que las nuevas empresas traduzcan sus planes de negocio y estrategias a acciones concretas para dar respuesta rápida y efectiva al dinamismo del entorno.

En este estudio se incluye al modelo la orientación al aprendizaje. Weerawardena, O'Cass y Julian (2006) han diferenciado tres tipos de orientación al aprendizaje: i) la orientación al aprendizaje de mercado; ii) la orientación al aprendizaje relacional; $y$, iii) la orientación al aprendizaje interno. De este último, algunos autores como Sinkula, Baker y Noordewier (1997) solo reconocen tres dimensiones: i) compromiso con el aprendizaje; ii) mentalidad abierta; y, iii) visión compartida, en tanto otros lo conciben con cuatro factores: compromiso con el aprendizaje, mentalidad abierta, visión compartida e intercambio de conocimiento. Baker y Sinkula (2002) han propuesto que la orientación al aprendizaje puede ser vista como el grado en el que la empre- 
sa cuestiona sus creencias y prácticas actuales para buscar proactivamente nuevo conocimiento que maximice el rendimiento de la organización. Para esta investigación, las definiciones más influyentes son las de Baker, Sinkula (2002) y las de Sinkula, Baker y Noordewier (1997).

Cardona y Calderón (2006) han señalado que la orientación al aprendizaje tiene las siguientes tres dimensiones: i) el compromiso con el aprendizaje; ii) la capacidad para desaprender lo aprendido; y, iii) la apertura y la experimentación.

Staples, Greenaway y McKeen (2001) han definido a la orientación al aprendizaje como la información que se convierte en conocimiento cuando interviene la inteligencia de las personas.

El conocimiento tiene una mayor riqueza para la organización. Para $\mathrm{Wu}$ (2007), las nacientes pymes deben desarrollar procesos que les permita obtener e internalizar nuevo conocimiento para lograr su supervivencia y crecimiento en el mercado. Son capacidades empresariales intangibles de difícil imitación, relevantes para el desarrollo de ventajas competitivas en las empresas.

\section{Antecedentes de la orientación al aprendizaje}

El estudio de Morgan y Strong (1998) confirma que las empresas con mayor grado de orientación al mercado muestran mayores capacidades de aprendizaje organizacional (en concreto: habilidades directivas, el proceso de desarrollo estratégico, la flexibilidad operativa y el conocimiento estratégico).

Santos et al. (2005) comprobaron el efecto de la orientación al aprendizaje como antecedente de la orientación al mercado operativo. Finalmente, Eusebio, Llonch y López (2004) y Llonch, López y Eusebio (2005) confirman que la orientación al aprendizaje es un antecedente de la orientación al mercado y que dicha relación está moderada por la procedencia del director general.

Para Sinkula, Baker y Noordewier (1997), la orientación al aprendizaje se refiere a las actividades que desarrolla la organización en la creación y difusión de conocimiento, para lograr resultados superiores y desarrollar ventajas competitivas. Esto incluye obtener y compartir información sobre necesidades actuales y futuras de los clientes, sobre los cambios en el mer- 
cado y sobre las acciones de los competidores, implica además la creación de estructuras organizacionales más eficientes, la optimización del uso de tecnología para reducir costos administrativos y el desarrollo de nuevos productos o servicios. Las definiciones de estos autores son las más influyentes.

\section{Consecuencias de la orientación al aprendizaje}

Esta orientación favorece a la sensibilidad de las empresas en la detección oportuna de los cambios en el comportamiento del mercado, lo cual es muy importante para las empresas.

Las organizaciones con mayor capacidad de aprender tienen altas posibilidades de creación de nuevo conocimiento; por ende, logran elevar su flexibilidad y responden con mayor velocidad que sus competidores a los cambios que se presentan en el entorno.

En este sentido, uno de los retos de las pymes ecuatorianas es la respuesta inmediata a las acciones de la competencia y la detección de las expectativas de los clientes.

De acuerdo con la evidencia empírica, existe una relación positiva directa entre la orientación al aprendizaje y los resultados de las organizaciones, tanto para las empresas grandes como para empresas ya establecidas en el mercado. Aunque también se encuentran trabajos de Hebel (2007) y Weerawardena, O’Cass y Julian (2006), donde la relación entre la orientación al aprendizaje y los resultados muestran poca o nula significación.

Debido a lo antes mencionado, como principal hipótesis para la presente investigación se puede expresar: los factores "orientación al mercado" y "orientación al aprendizaje" están relacionados y son innatos. La "orientación al aprendizaje" se ubica en medio de la relación "orientación al mercado-resultados organizacionales". Y los mejores beneficios se pueden obtener con un alto nivel de "orientación al mercado".

De acuerdo con la revisión de literatura, se estudian las hipótesis a continuación:

1. La orientación al mercado tiene un efecto directo y positivo con los resultados de las nuevas pequeñas y medianas industrias ecuatorianas.

2. La orientación al aprendizaje tiene un efecto directo y positivo con los resultados de las nuevas pequeñas y medianas industrias ecuatorianas. 
3. La orientación al mercado tiene un efecto directo y positivo con la orientación al aprendizaje de las nuevas pequeñas y medianas industrias ecuatorianas.

\section{Metodología}

Para cumplir con el objetivo general, contrastación de hipótesis y abordaje del estudio, el presente proyecto se basó en el método de investigación deductivo hipotético, de carácter transversal y cuantitativo. El modelo teórico y las hipótesis propuestas fueron contrastadas mediante la aplicación de análisis factorial confirmatorio de las escalas y un análisis basado en el modelo de ecuaciones estructurales. Se realizó un trabajo de campo por medio de encuestas a la población objetivo.

Tabla 1

Ficha técnica de la investigación

\begin{tabular}{|c|c|}
\hline Población objetivo: & $\begin{array}{l}\text { Empresas pequeñas y medianas } \\
\text { del sector de "Alimentos y Bebidas". }\end{array}$ \\
\hline Unidad muestral: & Propietario/director/fundador. \\
\hline Ámbito: & Provincia de Pichincha, Ecuador. \\
\hline Método de recogida de la información: & $\begin{array}{l}\text { Encuestas estructuradas proporcionadas } \\
\text { a los directivos a través de visitas físicas. }\end{array}$ \\
\hline Población $(N)$ & 390 \\
\hline Tamaño muestral $(n)$ & 194 \\
\hline Error muestral $(E)$ & $5 \%$ \\
\hline Nivel de confianza $(q)$ & $95 \% ; \theta=1,96 ; p=q=0,5$ \\
\hline Procedimiento de muestreo: & Discrecional. \\
\hline Fecha de trabajo de campo: & Entre marzo y septiembre de 2016. \\
\hline
\end{tabular}

Elaboración propia. 


\section{Desarrollo de escalas de medición}

\section{Diseño del cuestionario de la encuesta}

En las encuestas se utilizó el método de evaluación sumaria o escala Likert de siete niveles, donde "1" significa "totalmente en desacuerdo" y "7" significa "totalmente de acuerdo". A continuación, se presentan las variables y dimensiones para cada instrumento de medición.

\section{Escalas del instrumento de medición}

Para este estudio se ha considerado que el enfoque cultural de la escala de Narver y Slater (1990) es la que se adapta mejor a esta investigación basada en los recursos y capacidades, donde la variable cultural es un recurso valioso y de difícil imitación. No se ha tomado en cuenta el enfoque comportamental de la escala de Kohli y Jaworski (1993).

Tabla 2

Escalas de los instrumentos de medición

\begin{tabular}{|c|c|c|c|}
\hline Constructo & Tratamiento & Escala & Fuente \\
\hline \multirow{2}{*}{ Mercado } & \multirow{2}{*}{ Exógena } & 14 items (6/4/4); ordinal & \multirow{2}{*}{$\begin{array}{l}\text { Adaptado de la escala MKTOR } \\
\text { de Narver y Slater (1990). }\end{array}$} \\
\hline & & Likert-7; Independiente & \\
\hline \multirow{2}{*}{ Aprendizaje } & \multirow{2}{*}{ Endógena } & 13 ítems (5/4/4); ordinal & \multirow{2}{*}{$\begin{array}{c}\text { Sinkula, Baker y Noordewier } \\
\text { (1997). }\end{array}$} \\
\hline & & Likert-7; Independiente & \\
\hline \multirow[t]{2}{*}{ Emprendimiento } & \multirow[t]{2}{*}{ Endógena } & 6 ítems; ordinal & $\begin{array}{c}\text { Adaptado de la escala de Na- } \\
\text { man y Slevin (1993). }\end{array}$ \\
\hline & & Likert-7; Independiente & Origen de Covin y Slevin (1988) \\
\hline \multirow{2}{*}{ Innovación } & \multirow{2}{*}{ Endógena } & 3 items; ordinal & \multirow{2}{*}{ Baker y Sinkula (1999a). } \\
\hline & & Likert-7; Independiente & \\
\hline \multirow{2}{*}{ Resultados } & \multirow{2}{*}{ Endógena } & 6 ítems; ordinal & \multirow{2}{*}{$\begin{array}{l}\text { (Narver y Slater 1990; Jaworski } \\
\text { et al. 1993; Kirca et al. 2005) }\end{array}$} \\
\hline & & Likert-7; Independiente & \\
\hline
\end{tabular}

Elaboración propia. 
La encuesta de medición para la orientación al mercado es una adaptación de la escala de Narver y Slater (1990), la cual está basada en tres elementos: i) orientación al cliente; ii) orientación a la competencia; y, iii) coordinación interfuncional.

La encuesta de medición para la orientación al aprendizaje es una adaptación de la escala de Sinkula (1997), la cual está basada en tres elementos: i) compromiso con el aprendizaje; ii) mentalidad abierta; y, iii) visión compartida. Este cuestionario ha sido utilizado en varios trabajos, como mencionan Baker y Sinkula (2002) y Santos et al. (2005).

La encuesta de medición para los resultados organizacionales es una adaptación de las escalas de Jaworski y Kohli (1993), Narver y Slater (1990) y finalmente por Kirca, Jayachandran y Bearden (2005), ya que estas investigaciones han tomado en cuenta aspectos de satisfacción de clientes, empleados, operaciones y finanzas.

\section{Método estadístico para el análisis de datos}

A partir de los modelos de regresión, simple o múltiple, resultan insuficientes para las ciencias comportamentales y sociales, que tratan de conocer $\mathrm{y}$ entender una realidad compleja y multideterminada, y por lo tanto precisa explicaciones más amplias y complejas. Es necesario aplicar otros modelos, como las ecuaciones simultáneas, modelos multiecuacionales o modelos causales.

En este estudio, primeramente, se han validado las escalas de las medidas mediante análisis factorial confirmatorio (AFC), que permite evidenciar la validez del constructo.

Segundo, para verificar las hipótesis propuestas se ha empleado el modelo de ecuaciones estructurales o SEM (Structural Equation Model), una técnica de análisis multivariante para probar y estimar relaciones causales, a partir de datos estadísticos y asunciones cualitativas. 


\section{Análisis de fiabilidad}

En el estudio se incluyeron cuatro análisis:

1. Confirmar por un grupo de expertos la validez del contenido de las escalas.

2. Confirmar que no existan discrepancias en las respuestas a las encuestas.

3. Confirmar la consistencia de las escalas propuestas mediante análisis factorial.

4. Confirmar la confiabilidad de dichas escalas.

\section{Análisis factorial de los componentes de las escalas}

En el examen del sesgo potencial de no respuesta, se ha seguido el procedimiento sugerido por Armstrong y Overtone (1977). Los resultados obtenidos permiten afirmar que la muestra es representativa de la población objetivo de estudio de acuerdo con el procedimiento prueba t para muestras independientes.

\section{Análisis factorial de los componentes de las escalas}

Para cada análisis factorial: matriz de correlaciones de variables, incluidos niveles de significación, determinante, inversa; matriz de correlaciones reproducida; medida de la adecuación muestral KMO (Kaiser-Meyer-Olkin) y prueba de esfericidad de Bartlett.

La consistencia de todos los componentes de todas las escalas propuestas ha mostrado un valor superior al 0,70, mínimo admisible para el $\alpha$ (Nunnally 1978). Se utilizaron dos tipos de contrastes: a) test de esfericidad de Bartlett, que en el caso de tener un nivel de significación menor a 0,05 se puede decir que la estructura factorial subyacente a los datos es aceptable; y b) KMO, para la cual se ha recomendado que sea superior a 0,7 (Trespalacios, Vásquez y Bello 2005). 


\section{Fiabilidad de las escalas}

Las respuestas a las encuestas de los 194 propietarios de pequeñas y medianas industrias mostraron los valores de fiabilidad de las escalas orientadas al mercado y orientadas al aprendizaje. Con base en el coeficiente Alpha de Cronbach se calcularon las correlaciones de cada ítem, que dan como resultado coeficientes de relaciones positivas. Algo que se debe tomar en consideración es que el valor del $\alpha$ es el promedio de todos los coeficientes de correlación (Lind, Marchal y Wathen 2012).

Para la escala de orientación al mercado y sus tres componentes: a) orientación al cliente; b) orientación a la competencia; y, c) coordinación interfuncional han mostrado valores superiores al 0,80 de la muestra total. De acuerdo con Nunnally (1978), este valor es admisible en trabajos de investigación.

Para la escala orientación al aprendizaje y sus tres componentes: a) compromiso con el aprendizaje; b) visión compartida; y, c) mentalidad abierta muestran valores superiores al 0,9 de la muestra total y en los valores ítema-ítem valores superiores al 0,89. Dichos resultados son estadísticamente significativos.

\section{Validez convergente}

La validez convergente prueba que los constructos que se espera estén relacionados entre sí y la correlación entre ellos debe ser alta. Lehmann, Gupta y Steckel (1998) han mencionado que una medida tiene validez convergente si sigue el mismo modelo que las restantes medidas del concepto.

Para los dos constructos: orientación al mercado y orientación al aprendizaje, existe una correlación significativa de los tres componentes de cada una de las escalas, lo que evidencia que los elementos convergen en un constructo común, demostrando validez convergente (coeficientes de correlación significativas $(\mathrm{p}<0,01)$.

Hair, Anderson, Tathan y Black (1998) han recomendado que, además de ser significativos, el promedio de las cargas sobre cada factor sea superior a 0,7 en promedio. 


\section{Análisis factorial confirmatorio}

Dentro del análisis convergente, adicionalmente se utilizó el análisis factorial confirmatorio (AFC). Una vez formulada la hipótesis concreta sobre la relación entre indicadores y dimensiones latentes, se ha contrastado dicha hipótesis.

Adicionalmente, se ha comparado fiabilidad y validez del modelo de medida, se identificaron las relaciones causales entre la variable latente, mediante el análisis de relaciones causales, de manera que se pueda determinar la influencia directa o indirecta de cada una de las variables latentes en otras y cómo están relacionadas (Byrne 2001).

\section{Validez discriminante}

La validez discriminante permite evaluar las escalas de medida de las variables latentes. De este modo la validez de contenido actúa como un criterio robusto frente a determinados análisis estadísticos basados en covarianza.

Campbell y Fiske (1959) han afirmado que, para que unas medidas sean válidas, las de un mismo constructo deben correlacionarse altamente entre ellas (convergente) y que dicha correlación debe ser mayor que la que exista con respecto a las medidas propuestas para otro constructo distinto (discriminante). El análisis muestra correlación estadísticamente significativa de los ítems de cada elemento para con el factor de su componente.

\section{Análisis de datos y resultados}

Hipótesis (H) 1: La orientación al mercado tiene un efecto directo y positivo con los resultados de las pymes ecuatorianas.

$\mathrm{La} \mathrm{H}_{1}$ queda contrastada. Sí existe una relación efectiva entre la orientación al mercado y los resultados organizacionales en las pymes ecuatorianas, y es estadísticamente significativa: 0,731. Las investigaciones de Jaworski y Kholi (1993), Narver y Slater (1990) y Pelham (1999) presentan resultados similares en comparación con este estudio empírico. 
Hipótesis 2. La orientación al aprendizaje y los resultados tienen un efecto directo y positivo en las pymes ecuatorianas.

$\mathrm{La}_{2}$ no queda contrastada; no existe una relación efectiva entre la orientación al aprendizaje y los resultados organizacionales, estadísticamente significativa: 0,1. Las investigaciones de Hurley y Hult (1998) y Baker y Sinkula (1999) han coincidido también con este resultado.

La orientación al aprendizaje no tiene una relación efectiva si la organización no crea y comunica conocimiento; si no obtiene información de los clientes; si sus estructuras organizacionales no son eficientes; y lo más importante, si no desarrolla nuevos productos o servicios. Adicionalmente, la capacidad de adaptación, adaptabilidad y creatividad de dichas instituciones, las cuales buscan constantemente alternativas y soluciones para conquistar y mantener su lugar en el mercado.

De acuerdo con Sinkula (1997) y Baker y Sinkula (1999), la orientación al aprendizaje favorece la sensibilidad de las empresas en la detección oportuna de los cambios en el comportamiento del mercado.

La orientación al aprendizaje tiene un mayor impacto en los resultados organizacionales cuando la empresa primero trabaja en una orientación al mercado. Las compañías con mayor capacidad de aprender tienen altas posibilidades de creación de nuevo conocimiento, por ende, logran elevar su flexibilidad y responden con mayor velocidad que sus competidores a los cambios que se presentan en el entorno.

Santos et al. (2005) han comprobado el efecto de la orientación al aprendizaje como antecedente de la orientación al mercado. Finalmente, Eusebio, Llonch y López (2004) y Llonch, López y Eusebio (2005) confirmaron que la orientación al aprendizaje es un antecedente de la orientación al mercado y que esta relación está moderada por la procedencia del director general.

Hipótesis 3. La orientación al mercado tiene un efecto directo y positivo con la orientación al aprendizaje de las pymes ecuatorianas.

$\mathrm{La} \mathrm{H}_{3}$ queda contrastada. Sí existe una relación efectiva entre la orientación al mercado y la orientación al aprendizaje estadísticamente significativa: 0,8 . La orientación al aprendizaje tiene como antecedente la orientación al mercado.

Santos et al. (2005) ha comprobado el efecto de la orientación al aprendizaje como antecedente de la orientación al mercado. Finalmente, Eusebio, 
Llonch, y López (2004) y Llonch, López y Eusebio (2005) confirmaron que la orientación al aprendizaje es un antecedente de la orientación al mercado y que esta relación está moderada por la procedencia del director general.

\section{Discusión}

Con la evidencia empírica se confirma que el presente estudio coincide con la invetigación de Kohli y Jaworski (1990) y Narver y Slater (1990), donde la orientación al mercado permite a las organizaciones adaptarse a las necesidades y requerimientos del mercado, pero la orientación al aprendizaje (Sinkula, Baker y Noordewier 1997) promueve que las empresas actúen proactivamente y facilita la capacidad innovadora de manera constante. Los resultados organizacionales (Jaworski y Kohli 1993; Narver y Slater 1990; Kirka, Jayachandran y Bearde 2005) también han demostrado que el impacto de la orientación al mercado y el aprendizaje organizacional sobre el rendimiento está influido por la innovación (Hurley y Hult 1998; Hult, Ketchen 2001) y lo orientados que están al emprendimiento (Naman y Slevin 1993).

Se ha demostrado que sí existe una relación efectiva entre la orientación al mercado y los resultados organizacionales en las empresas ecuatorianas, de acuerdo con las definiciones más influyentes de los autores Kohli y Jaworski (1990) y Narver y Slater (1990), estudios empíricos que coinciden. Esto es, cuando las empresas recogen la información de las necesidades de los clientes actuales y futuros (subdimensión: orientación al cliente), la comunicación hacia todos los colaboradores de la organización, llamada subdimensión "coordinación interfuncional"; analizar y discutir las estrategias de los competidores (subdimensión: orientación a la competencia) no para imitarlas, sino para diseñar estrategias diferentes. Estas subdimensiones que conforman la orientación al mercado en su conjunto, permiten tener una cultura organizacional que crea un valor superior para los clientes o consumidores, como consecuencia, un mejor resultado en los beneficios empresariales.

Esta primera hipótesis tiene un mayor impacto cuando la empresa toma en cuenta al entorno externo, esto significa que debe estar atenta al crecimiento del sector, al grado de competencia, al cambio tecnológico en los productos/servicios que se ofertan y al grado de cambio en las preferencias 
de los clientes. Este estudio empírico coincide con los resultados de Kohli y Jaworski (1990), que han demostrado que no solamente se requiere conocer sobre el cliente, sino que también se requieren años para que la organización desarrolle productos/servicios de valor para el consumidor.

En el estudio realizado sobre la orientación al mercado, todavía se puede observar que las pymes ecuatorianas, poseen un tipo de cultura organizacional que tiene como meta satisfacer a los clientes actuales y potenciales. Pero estas empresas todavía no comprenden del todo las actividades de coordinación interfuncional y orientación a la competencia. De acuerdo con esta investigación, se sugiere que las direcciones de las empresas desarrollen su actividad de comunicación interna y también implementen estrategias diferenciadoras, ya sea creando y promocionando una categoría diferente para productos genéricos o incrementando el grado de diferenciación en sus productos/servicios.

En cuanto a la segunda hipótesis, no se demostró que exista una relación efectiva de la orientación al aprendizaje y los resultados de la pyme ecuatoriana.

Santos et al. (2005) ha comprobado el efecto de la orientación al aprendizaje como antecedente de la orientación al mercado. Finalmente, Eusebio, Llonch y López (2004) y Llonch, López y Eusebio (2005) han confirmado que la orientación al aprendizaje es un antecedente de la orientación al mercado y que esta relación está moderada por la procedencia del director general.

De acuerdo con Sinkula (1997), la orientación al aprendizaje favorece la sensibilidad de las empresas en la detección oportuna de los cambios en el comportamiento del mercado.

De acuerdo con el estudio empírico del presente trabajo y también a las definiciones más importantes e influyentes de Hurley y Hult (1998), Baker y Sinkula (1999), Farrell (2000) y Morgan y Strong (1998), el constructo propuesto en esta investigación tendría un impacto efectivo o positivo en los resultados cuando las empresas ecuatorianas trabajen con un mayor grado de orientación al mercado y sus tres subdimensiones (ver también las conclusiones de la tercera hipótesis). Las empresas con mayor capacidad de aprender tienen altas posibilidades de creación de nuevo conocimiento, por ende, elevan su flexibilidad y responden con mayor velocidad que sus competidores a los cambios que se presentan en el entorno. Esta afirmación está basada 
en los resultados del estudio empírico y coincide con el modelo de Hurley y Hult (1998), Baker y Sinkula (1999) y Sinkula (1994).

Otra razón por la que la hipótesis no fue contrastada, se debe a los antecedentes relacionados con el aprendizaje organizacional. El estudio de Morgan y Strong (1998) confirma que las empresas con mayor grado de orientación al mercado muestran mayores capacidades de aprendizaje organizacional. Adicionalmente, la variable de control "Dinamismo del Entorno" tiene influencia nula en la relación directa entre orientación al aprendizaje y los resultados organizacionales. Por este motivo, la hipótesis no queda contrastada.

La tercera hipótesis demuestra que sí existe una relación efectiva entre la orientación al mercado y la orientación al aprendizaje, es decir, cuando las empresas ecuatorianas explotan las oportunidades del entorno a través del aprendizaje en la organización, pero requieren previamente de la identificación de dichas oportunidades con base en el conocimiento que ofrece la orientación al mercado. Se coincide con las definiciones de Slater y Narver (1995); Hurley y Hult (1998); Sinkula (1994); Sinkula, Baker y Noordewier (1997); y Covin y Slevin (1990).

Se coincide con el segundo modelo: (orientación al mercado tiene relación directa con orientación al aprendizaje $=$ resultados organizacionales). El presente estudio empírico coincide con dicho modelo alternativo $(\mathrm{OM} \rightarrow \mathrm{OA})$ de Slater y Narver (1996) y de Hurley y Hult (1998).

OA $\rightarrow$ OM $\rightarrow$ resultados (Day 1994; Farrel y Oczkowski 2002; Santos y Cols 2015).

OM $\rightarrow$ OA $\rightarrow$ resultados (Slater y Narver 1995; Hurley y Hult 1998).

$\mathrm{OM} \rightarrow \mathrm{OA} \rightarrow$ resultados (Baker y Sinkula 1999; Farrel 2000; Morgan 2004).

La orientación al aprendizaje y la orientación al mercado influyen en la consecución de mejores resultados organizacionales. Esta línea argumental sugiere que la orientación al mercado presenta una serie de limitaciones a la hora de conseguir ventajas competitivas y que, por tanto, no es suficiente estar orientado al mercado, sino que las empresas tienen mayores capacidades competitivas si presentan al mismo tiempo una fuerte orientación al mercado y una fuerte orientación al aprendizaje. Es decir, que una orientación al aprendizaje fomenta la orientación al mercado y que ambas orientaciones 
desembocan en la obtención de unos mejores resultados. Así, el presente estudio concuerda con Sinkula (1994); Sinkula, Baker y Noordewier (1997); y Morgan, Katsikeas y Appiah-Adu (1998).

\section{Conclusiones}

Se confirmaron las relaciones positivas de las tres variables en el modelo teórico, mediante el estudio explicativo. Los factores "orientación al mercado" y "orientación al aprendizaje" están relacionados y son innatos. La "orientación al aprendizaje" se ubica en medio de la relación "orientación al mercado-resultados organizacionales" y los mejores beneficios se pueden obtener con un alto nivel de "orientación al mercado", ya que cuando el esfuerzo de la organización es bajo, se desperdician oportunidades de mercado y la excesiva inversión en innovación de productos/servicios no se ve recompensada con los beneficios deseados.

Adicionalmente, se confirma que sí existe un vínculo entre la orientación al mercado y aprendizaje, lo cual se traduce en resultados organizacionales efectivos, que si bien no alcanzan niveles significativos en el desarrollo de las nuevas pymes ecuatorianas, no debe sustentarse dicho resultado en falta o deficiencia de creatividad e iniciativa personal, sino en la actual cultura empresarial existente, la cual debe priorizar estudios de mercado y actividades de desarrollo investigativo de nuevos productos/servicios.

Esta información es un ingrediente crítico e invaluable para apoyar la toma de decisiones del empresario, porque el emprendedor requiere dar respuesta rápida a las necesidades de sus clientes. Este modelo conceptual está ajustado a sus peculiaridades.

Las contribuciones del estudio han sido: i) confirmar la relación efectiva de la orientación al mercado y la orientación al aprendizaje, sobre los resultados en las pymes ecuatorianas; y, ii) confirmar que la orientación al mercado permite a las organizaciones adaptarse a las necesidades y requerimientos del mercado, pero la orientación al aprendizaje promueve que las empresas actúen proactivamente y facilita la capacidad innovadora de manera constante. 


\section{Referencias}

Armstrong, Scott, y Terry Overtone. 1977. "Estimating Nonresponse Bias in Mail surveys". Journal of Marketing Research 14 (1): 396-402. 10.2307/3150783.

Baker, William, y James Sinkula. 1999. "Learning Orientation, Market Orientation, and Innovation: Integrating and Extending Models of Organizational Performance". Journal of Market Focused Management 4 (4): 295-308. https:// doi.org/10.1023/A\%3A1009830402395.

---. 1999. "The Synergistic Effect of Market Orientation and Learning Orientation on Organizational Performance". Journal of the Academy of Marketing Science 27 (4): 411-427. https:// doi.org/10.1177/0092070399274002.

---. 2002. "Market Orientation, Learning Orientation and Product Innovation: Delving into the Organization's Black Box". Journal of Market Focused Management 5 (1): 5-23. https:// doi.org/10.1023/A:1012543911149.

Byrne, Barbara. 2001. Structural Equation Modeling With AMOS: Basic Concepts, Applications, and Programming. NuevaYork: Routledge.

Campbell, Richard, y Donald Fiske. 1959. "Convergent and Discriminant Validation by the Multitrait-Multimethod Matrix". Psychol Bull 2 (56): 81-105. https://psycnet.apa.org/ doi/10.1037/h0046016.

Cardona, Alberto, y Gregorio Calderón. 2006. "El impacto del aprendizaje en el rendimiento de las organizaciones". Cuadernos de Administración 2 (19): 11-43. https://bit.ly/2ZWM TGm.

Covin, Jeffrey, Dennis Slevin y Teresa Covin. 1990. "Content and Performance of Growth Seeking Strategies: A Comparison of Small Firms in High and Low Technology Industries". Journal of Business Venturing 5 (6): 391-412. https://doi.org/10.1016/0883-90 26(90)90013-J.

Cronbach, Lee. 1951. "Coefficient Alpha and The Internal Structure of Tests". Psychometrika (16): 297-334. https://doi.org/10.1007/BF02310555.

Eusebio, Rossano, Joan Llonch y Pilar López Belbeze. 2004. "Learning Orientation and Market Orientation: Moderating Effects and Consequences". 33rd European Marketing Academy Conference (EMAC). Murcia 5: 18-21.

Farrell, Madi. 2000. "Developing a Market-Oriented Learning Organisation". Australian Journal of Management 25 (2): 201-222. https://doi.org/10.1177\%2F031289620002500205.

Farrell, Madi, y Edward Oczkowski. 2002. "Are Market Orientation and Learning Orientation Necessary for Superior Organizational Performance?”. Journal of Market Focused Management 5: 197-217.

Hair, Joseph, Rolph Anderson, Ronald Tatham y William Black. 1998. Multivariate Data Analysis. Nueva Jersey: Prentice Hall.

Hebel, Misha. 2007. "Light Bulbs and Change: Systems Thinking and Organizational Learning for New Ventures”. The Learning Organization 14 (6): 499-509. http://dx.doi.org/ 10.1108/09696470710825114. 
Hult, Thomas, David Ketchen y Stanley Slater. 2001. "Does Market Orientation Matter? A Test of the Relationship between Positional Advantage and Performance". Strategic Management Journal 22 (9): 899-906. https://doi.org/10.1002/smj.197.

Hult, Thomas, Robert Hurley y Gary Knight. 2004. "Innovativeness: Its Antecedents and Impact on Business Performance". Industrial Marketing Management 33 (5): 429-438. https://doi.org/10.1016/j.indmarman.2003.08.015.

Hunt, Shelby, y Robert Morgan. 1995. "The Comparative Advantage Theory of Competition”. Journal of Marketing 59 (2): 1-15. https://doi.org/10.1177\%2F002224299505900201.

Hurley, Robert, y Thomas Hult. 1998. "Innovation, Market Orientation and Organizational Learning: An Integration and Empirical Examination”. Journal of Marketing 62 (3): 42 54. https://www.jstor.org/stable/1251742.

Jácome, Hugo, y Katiuska King. 2013. Estudios industriales de la micro, pequeña y mediana empresa. Quito: FLACSO, Ecuador.

Jaworski, Bernard, y Ajay Kohli. 1993. "Market-Driven Versus Driving Market”. Journal of the Academy of Marketing Science 28 (1): 45-54. https://doi.org/10.1177/0092070300281005.

Kantis, Hugo, Masahiki Ishida y Masahiko Komori. 2002. "Empresarialidad en economías emergentes: creación y desarrollo de nuevas empresas en América Latina y el este de Asia: resumen". Buenos Aires: Banco Interamericano de Desarrollo. Accedido marzo de 2017. https://bit.ly/2XrpWsj.

Kirca, Ahmet H., Satish Jayachandran y William O. Bearden. 2005. "Market Orientation: A Meta-Analytic Review and Assessment of Its Antecedents and Impact on Performance". Journal of Marketing 69 (2): 24-41. www.jstor.org/stable/30162043.

Kohli, Ajay, y Bernard Jaworski. 1990. "Market Orientation: The Construct, Research Propositions and Managerial Implications". Journal of Marketing 54 (2): 1-18. https://psycnet. apa.org/doi/10.2307/1251866.

Lehmann, Donald R., Sunil Gupta y Joel H. Steckel. 1998. Marketing Research. Reading: Addison-Wesley.

Lind, Douglas, William Marchal y Samuel Wathen. 2012. Statistical Technics in Business \& Economics. Ciudad de México: McGraw-Hill.

Llonch, Joan, Pilar López Belbeze y Rossano Eusebio. 2005. "La relación entre la orientación al aprendizaje y la orientación al mercado". XI Congreso Nacional de ACEDE. San Cristóbal de la Laguna. Accedido febrero de 2017. http://www.acede.org.

Morgan, Robert, Constantine Katsikeas y Kwaku Appiah-Adu. 1998. "Market Orientation and Organizational Learning Capabilities". Journal of Marketing Management 14: 353381. 10.1362/026725798784959444.

Morgan, Robert, y Carolyn Strong. 1998. "Market Orientation and Dimensions of Strategic Orientation". European Journal of Marketing 32 (11/12): 1051-1073. https://doi. org/10.1108/03090569810243712. 


\section{Gustavo Gallo-Mendoza}

Naman, Jhon, y Dennis Slevin. 1993. "Entrepreneurship and The Concept of Fit: A Model and Empirical Test”. Strategic Management Journal 14: 137-153. http://dx.doi.org/10.21043/ equilibrium.v1i1.204.

Narver, Jhon, y Stanley Slater. 1990. "The Effect of a Market Orientation on Business Profitability”. Journal of Marketing 54 (4): 20-35. http://dx.doi.org/10.2307/1251757.

Nunnally, Jum. 1978. Psychometric Theory. Nueva York: McGraw-Hill.

Pelham, Al. 1999. "Influence of Environment, Strategy, and Market Orientation on Performance in Small Manufacturing Firms". Journal of Business Research 45 (1): 33-46. https: //doi.org/10.1016/S0148-2963(98)00026-5.

Santos, María Leticia, María José Sanzo, Luis Ignacio Álvarez y Rodolfo Vázquez. 2005. "Effects of Market Orientation on Business Strategic Behavior". Journal of Strategic Marketing 13: 17-42. 10.1080/0965254042000328677.

---. 2005. "Organizational Learning and Market Orientation: Interface and Effects on Performance”. Industrial Marketing Management 34: 187-202. https://oi.org/10.1016/j.indmarman. 2004.08.004.

Sinkula, Joe. 1994. "Market Information Processing and Organizational Learning”. Journal of Marketing 58 (1): 35-45. https://doi.org/10.1177/0092070397254003.

Sinkula, Joe, William Baker y Thomas Noordewier. 1997. “A Framework for Market-based Organizational Learning: Linking Values, Knowledge and Behavior". Journal of the Academy of Marketing Science 25: 305-318. https://doi.org/10.1177/0092070397254003.

Slater, Stanley. 1995. “Issues in Conducting Marketing Strategy Research”. Journal of Strategic Marketing 3 (4): 257-270. https://doi.org/10.1080/09652549500000016.

Slater, Stanley, y Jhon Narver. 1995. "Market Orientation and The Learning Organization". Journal of Marketing 59 (3): 63-74. https://doi.org/10.1177/002224299505900306.

---. 1996. "Marketing's Contribution to the Implementation of Business Strategy: An Empirical Analysis". Strategic Management Journal 22: 1055-1067. https://doi.org/10.1002/smj.198.

Staples, Sandy, Kathleen Greenaway y James McKeen. 2001. “Opportunities of Research About Managing the Knowledge-Based Enterprise". International Journal of Marketing Reviews (3): 1-20. https://doi.org/10.1111/1468-2370.00051.

Tinajero, Fernando. 2015. El emprendimiento ecuatoriano. Quito: El Conejo.

Trespalacios, Juan, Rodolfo Vázquez y Laurentino Bello. 2005. Investigación de mercados: métodos de recogida y análisis de la información para la toma de decisiones de marketing. Madrid: Ediciones Paraninfo.

Weerawardena, Jay, Aron O’Cass y Craig Julian. 2006. "Does Industry Matter? Examining the Role of Industry Structure and Organizational Learning in Innovation and Brand Performance". Journal of Business Research (59): 37-45. https://doi.org/10.1016/j.jbusres. 2005.02.004.

Wu, Lei-Yu. 2007. "Entrepreneurial Resources, Dynamic Capabilities and Start-Up Performance of Taiwan's High-Tech Firms". Journal of Business Research (60): 549-555. https: //doi.org/10.1016/j.jbusres.2007.01.007. 